\title{
Direct Immunoassays and Their Performance: Theoretical Modelling of the Effects of Antibody Orientation and Associated Kinetics
}

\author{
Dana Mackey \\ Technological University Dublin, dana.mackey@tudublin.ie \\ Eilis Kelly \\ Technological University Dublin \\ Robert Nooney \\ Dublin City University
}

See next page for additional authors

Follow this and additional works at: https://arrow.tudublin.ie/scschmatart

Part of the Applied Mathematics Commons, and the Mathematics Commons

\section{Recommended Citation}

Mackey, D., Kelly, E., Nooney, R. \& O'Kennedy, R. (2018). Direct immunoassays and their performance theoretical modelling of the effects of antibody orientation and associated kinetics. Integrative Biology, 2018,10, pp.598-604. doi:10.1039/C8IB00077H

This Article is brought to you for free and open access by the School of Mathematics at ARROW@TU Dublin. It has been accepted for inclusion in Articles by an authorized administrator of ARROW@TU Dublin. For more information, please contact arrow.admin@tudublin.ie, aisling.coyne@tudublin.ie,gerard.connolly@tudublin.ie.

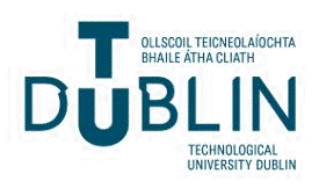




\section{Authors}

Dana Mackey, Eilis Kelly, Robert Nooney, and Richard O'Kennedy 


\title{
Direct immunoassays and their performance - theoretical modelling of the effects of antibody orientation and associated kinetics
}

\author{
D. Mackey, E. Kelly, \\ School of Mathematical Sciences \\ Dublin Institute of Technology \\ Kevin Street, Dublin 8, Ireland \\ R. NoOney And R.O'Kennedy \\ Biomedical Diagnostics Institute \\ Dublin City University \\ Glasnevin, Dublin 9, Ireland
}

\begin{abstract}
The orientation and activity of antibodies immobilized on solid surfaces are of direct relevance to many immunosensing applications. We therefore investigate a mathematical model which estimates the fraction of antibodies which are available for reaction in a randomly adsorbed sample. Numerical simulations are presented which highlight the separate effects of antibody orientation, accessibility and loss of binding ability on the amount of captured antigen. The assay response can then be expressed as a function of total antibody density and used for optimizing the surface coverage strategy under various conditions.
\end{abstract}

\section{Introduction}

Direct immunoassays are well established methods for detecting a variety of analytes. However, in many cases the formatting and the development of the assay are performed by carrying out a series of experiments where the key components (including antibodies, antigens, blocking agents etc.) are assessed to determine the ideal combinations that yield the best performance characteristics. In some cases this is based purely on a set of iterative serial dilutions of components in combination with fixed concentrations of one component, but the exact selection of optimised parameters is often relatively poorly defined. In an attempt to develop a more systematic approach to this procedure, we are proposing a theoretical model which investigates the effect of many design parameters on the kinetics of a direct immunoassay, as well as their relative importance under various conditions.

Many immunoassay technologies rely on the immobilization of detection antibodies on solid interfaces for binding analytical targets such as pathogenic organisms, hormones or protein biomarkers for cardiovascular disease or cancer diagnostics. The most frequently used immobilization strategies are physical or chemical adsorption, both of which result in random 
orientation on the substrate and considerably reduced antigen-binding activity. This phenomenon, generally attributed to shielding of antibody active sites and molecule denaturation upon contact with the surface, has been widely researched and described in the experimental literature. Some studies, [1,2], report that extremely small (typically less that 10\%) fractions of antibodies are still capable of binding antigen after immobilization. In addition, the functionality of immobilized antibodies is of great importance to other biomedical applications (such as artificial tissues and implants or targeted drug delivery systems) as well as immune reactions in vivo [3].

The amount of captured antigen has been studied as a function of antibody surface coverage both theoretically and experimentally by several authors $[1,2,4,3]$. However, since the density of adsorbed antibodies is known to affect their orientation, accessibility and binding ability, it is often not clear which of these properties has a greater impact on the assay signal, and their separate assessment can be difficult to achieve in practice [5]. The mathematical model we are proposing aims to address this issue.

Our modelling strategy is based on the theory known as Random Sequential Adsorption (RSA), which has been successfully used over the past few decades for describing monolayer particle deposition in many physical and biological settings, $[6,7]$. In the most standard description, uniform particles are randomly and irreversibly placed onto a solid surface with no overlap allowed (although generalized theories exist which remove these restrictions). The process is continued until no more particles can be deposited and the so-called jamming limit is reached. The RSA process in one dimension is referred to as the interval filling, or car parking, problem and the jamming coverage (also known as the Rényi constant) was calculated in [8] as $C_{R} \approx 0.74756 \ldots$ This theory can be used for calculating the distribution of adsorbed particles, as well as the gaps between them, at any moment during the deposition process.

In addition, we have recently presented a mathematical model for quantifying the orientation and activity of antibodies immobilized on a solid surface, based on the RSA framework [9]. In this model, the antibodies were represented by randomly oriented circles whose diameters cover a 1-dimensional substrate and a simple procedure was developed for estimating the percentage of antibodies which allow antigen access out of total antibody coverage. The obvious disadvantages of a one-dimensional formulation are the difficulties in describing all orientation configurations and comparing essential model parameters (such as concentrations, kinetic rate constants, etc.) to their experimentally determined counterparts; however, a thorough understanding of this basic model is essential before generalizations to higher dimensions and more realistic physical problems can be constructed. In spite of its restrictive setting, this modelling strategy produced promising results and showed good qualitative agreement with previous experimental studies, $[9,10]$. In particular, it reproduced the signal drop at high surface densities under similar conditions to those reported in the literature, $[1,2,11]$, which indicates the possibility of a well-defined optimal antibody coverage (with obvious implications for design considerations).

The current paper generalizes our previous model (which assumed uniform kinetic activity) to address the more realistic situation where the ability of antibodies to bind antigen, as described by the equilibrium dissociation constant $\left(K_{d}\right)$, depends on their immobilization configuration. Experimental evidence suggests that the interaction with the solid surface has a detrimental effect on the antibody activity and so we expect upright antibodies (which have less contact) to retain higher binding ability than the ones lying flat on the substrate. This generalization allows us to study antibody orientation and affinity separately and, through 
extensive numerical simulations, to assess their relative importance in a variety of cases.

While the proposed model has some limitations, we believe it can offer new approaches and insights into optimization of assay design. For example, the RSA methods outlined above allow us to further differentiate between orientation and accessibility by investigating the spacing between active sites of adjacent antibodies. Although the effect of antibody orientation on antigen capturing activity has been widely investigated in the literature $[1,2$, $5,17,18$, the antibody gap distribution throughout the adsorption process is not a frequently used parameter in assay optimization studies.

\section{Mathematical model}

\subsection{Derivation of active coverage}

The standard, one-dimensional RSA formulation assumes that equal size intervals are placed randomly and sequentially at a fixed rate onto an infinite line, provided they do not overlap already deposited intervals. The length of these intervals is irrelevant to the analysis but, for convenience, it is usually assumed to be equal to 1 . The kinetic approach detailed in [12] introduces a gap density function $P(x, t)$, which measures the number of gaps (empty spaces between adsorbed particles) of length $x$ at time $t$ and can be calculated as follows,

$$
P(x, t)=\left\{\begin{array}{ll}
t^{2} F(t) \mathrm{e}^{-(x-1) t}, & \text { if } x \geq 1 \\
2 \int_{0}^{t} \tau F(\tau) \mathrm{e}^{-x \tau} d \tau, & \text { if } x<1
\end{array},\right.
$$

where

$$
F(t)=\exp \left[-2 \int_{0}^{t} \frac{1-\mathrm{e}^{-u}}{u} d u\right],
$$

(see also $[7,9]$ ). The total coverage function is given by

$$
\theta(t)=1-\int_{0}^{\infty} x P(x, t) d x=\int_{0}^{t} F(\tau) d \tau,
$$

a non-dimensional quantity which represents the fraction of the line (or substrate) covered by intervals (particles) at time $t$ and converges in the long term to the jamming limit, or Rényi's constant,

$$
\lim _{t \rightarrow \infty} \theta(t)=C_{R}=0.74756 \ldots
$$

Antibodies are large Y-shaped molecules, comprised of two fragment antigen binding (Fab) regions and a fragment crystallizable region $(\mathrm{Fc})$. In random (non-directed) immobilization, antibody orientation is usually described (see, for example, $[2,13,14]$ ) by one of the following positions: "end on" (the ideal position, with the active Fab region pointing towards the solution), "head on" (or Fab down), "flat on" and "side on" (both of which correspond, in our 1-dimensional model, to a side orientation, as shown in Fig. 1). As mentioned in the Introduction, a simple procedure was introduced in [9] for estimating the "active coverage", that is the fraction of antibodies whose Fab sites were accessible to antigens in solution, out of total antibody coverage. The assumption made in this model was that an end-on ("Up") antibody is unconditionally accessible, while a side-oriented antibody is accessible provided there is a gap of length at least $\delta$ between its binding site and the adjacent adsorbed particle (where the parameter $\delta$ is related to the size of the antigen of interest). The head-on 

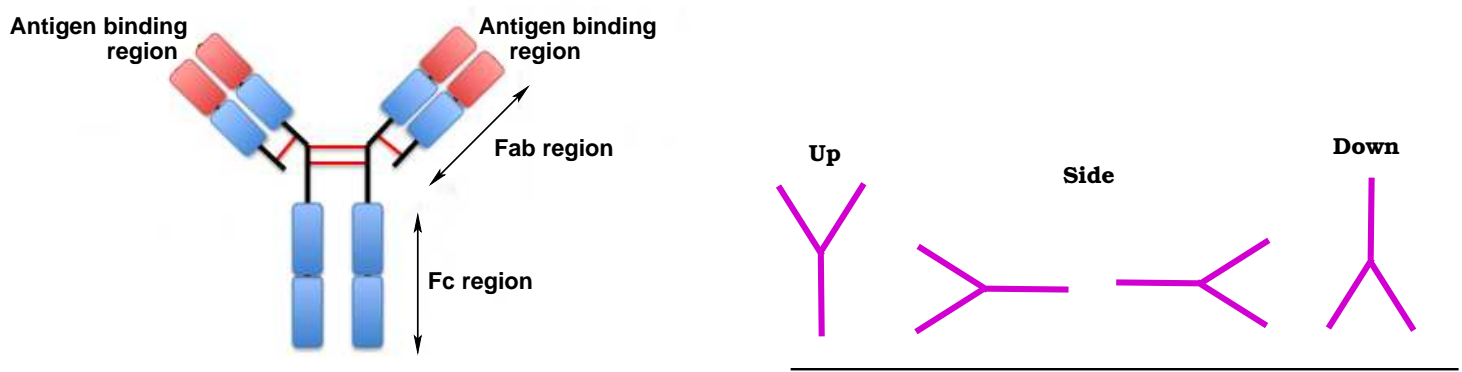

Figure 1: Antibody structure and immobilization orientations. In our 1-dimensional description, an antibody can adsorb either "Up" (end-on), "Down" (head-on) or "Side" (flat-on or side-on).

("Down") antibodies are considered inaccessible. The active coverage can then be written as $\mathcal{B}(t)=\mathcal{B}_{u}(t)+\mathcal{B}_{s}(t)$, where $\mathcal{B}_{u}(t), \mathcal{B}_{s}(t)$, are coverages by "up" (end-on) and accessible side-on antibodies, respectively, and were calculated (using the gap density function) as, [9],

$$
\begin{aligned}
\mathcal{B}_{u}(t) & =P_{\text {up }} \theta(t), \\
\mathcal{B}_{s}(t) & =2 P_{\text {side }}\left(t F(t)+2 \int_{0}^{t}\left(\mathrm{e}^{-\delta \tau}-\mathrm{e}^{-\tau}\right) F(\tau) d \tau\right) \\
- & P_{\text {side }}^{2} \times\left\{\begin{array}{cc}
2 \int_{0}^{t}\left(\mathrm{e}^{-\delta \tau}-\mathrm{e}^{-2 \delta \tau}\right) F(\tau) d \tau, & \text { if } 2 \delta<1 \\
t F(t)\left(1-\mathrm{e}^{-(2 \delta-1) t}\right) & \\
+2 \int_{0}^{t}\left(\mathrm{e}^{-\delta \tau}-\mathrm{e}^{-\tau}\right) F(\tau) d \tau, & \text { if } 2 \delta \geq 1
\end{array}\right.
\end{aligned}
$$

where $P_{\text {up }}, P_{\text {side }}$ are the percentages of particles in the corresponding orientations. We normalize the active and total coverages by defining

$$
\overline{\mathcal{B}}=\frac{\mathcal{B}}{C_{R}}, \quad \bar{\theta}=\frac{\theta}{C_{R}},
$$

so that

$$
0 \leq \overline{\mathcal{B}}(t) \leq 1, \quad 0 \leq \bar{\theta}(t) \leq 1,
$$

for all $t$.

Note that this calculation simplifies the particle geometry by assuming that its width is equal to its height. A more general approach to estimating active surface coverage was given in [10], where the end-on and side-on configurations occupy intervals of different sizes and the antibody aspect ratio is introduced in the analysis as yet another parameter which might influence the assay behaviour.

\subsection{Antibody-antigen reaction kinetics}

If we consider the end-on and side-on antibodies as different species, with concentrations denoted by $B_{u}$ and $B_{s}$, and possibly different kinetic abilities, we can write the following reactions:

$$
A+B_{u} \underset{k_{u}^{\prime}}{\stackrel{k_{u}}{\rightleftarrows}} C_{u} \quad A+B_{s} \underset{k_{u}^{\prime}}{\stackrel{k_{u}}{\rightleftarrows}} C_{s},
$$


where $A$ is the antigen concentration, $C_{u}$ and $C_{s}$ are concentrations of bound antigen, while $k_{u}, k_{s}$ and $k_{u}^{\prime}, k_{s}^{\prime}$ are the association and dissociation rate constants, respectively. The reaction rates are then given by the differential equations

$$
\begin{aligned}
& \frac{d C_{u}}{d t}=k_{u} A(t)\left(B_{u}^{0}-C_{u}(t)\right)-k_{u}^{\prime} C_{u}(t), \\
& \frac{d C_{s}}{d t}=k_{s} A(t)\left(B_{s}^{0}-C_{s}(t)\right)-k_{s}^{\prime} C_{s}(t),
\end{aligned}
$$

where all concentrations are functions of time and $B_{s}^{0}, B_{u}^{0}$ are the initial concentrations of immobilized antibody. We also let

$$
K_{d}^{u}=\frac{k_{u}^{\prime}}{k_{u}}, \quad K_{d}^{s}=\frac{k_{s}^{\prime}}{k_{s}}
$$

be the equilibrium dissociation constants for the two antibody species, commonly used as a measure of binding affinity. (Recall that high $K_{d}$ values imply low affinity and vice-versa.)

The equilibrium condition is $\frac{d C_{u}}{d t}=\frac{d C_{s}}{d t}=0$, and we find that the steady-state values of the reaction products $C_{u}$ and $C_{s}$ satisfy the coupled equations

$$
\begin{aligned}
& C_{u}=\frac{C_{s} K_{d}^{s} B_{u}^{0}}{C_{s} K_{d}^{s}+K_{d}^{u}\left(B_{s}^{0}-C_{s}\right)}, \\
& \left(B_{s}^{0}-C_{s}\right)\left(A^{0}-C_{u}-C_{s}\right)-K_{d}^{s} C_{s}=0
\end{aligned}
$$

where $A^{0}$ is initial antigen concentration and we used the conservation law $A(t)+C_{u}(t)+$ $C_{s}(t)=A^{0}$. In order to make use of the RSA results discussed in Section 2.1, we must relate the initial active antibody concentrations to coverages as follows,

$$
B_{u, s}^{0}=\frac{\mathcal{B}_{u, s}}{D}=\frac{\overline{\mathcal{B}}_{u, s} C_{R}}{D} \equiv \kappa \overline{\mathcal{B}}_{u, s},
$$

where $D$ is the diameter (or length, in our 1-dimensional model) of the antibody molecule, while $\mathcal{B}_{u, s}$ are the initial active coverages, as calculated by (2)-(3). For the purpose of our theoretical model it is simpler to express concentrations as number of molecules per unit length (which is equivalent to the concept of molar concentration). The constant $\kappa=C_{R} / D$ can then be interpreted as the concentration of antibodies corresponding to the jamming coverage $C_{R}$.

The use of scaled (or non-dimensional) variables greatly facilitates the analysis and numerical simulations of mathematical equations and also allows them to be viewed in a more general context. For example, $\bar{\theta}=1$ refers to maximum (jamming) coverage, a concept common to any adsorption model (even though the jamming values for generalized RSA frameworks are different). Thus, the non-dimensional form of equations (5)-(6) become

$$
\begin{aligned}
& \mathcal{C}_{u}=\frac{\mathcal{C}_{s} \psi_{s} \overline{\mathcal{B}}_{u}}{\mathcal{C}_{s} \psi_{s}+\psi_{u}\left(\overline{\mathcal{B}}_{s}-\mathcal{C}_{s}\right)}, \\
& \left(\overline{\mathcal{B}}_{s}-\mathcal{C}_{s}\right)\left(\alpha-\mathcal{C}_{u}-\mathcal{C}_{s}\right)-\psi_{s} \mathcal{C}_{s}=0
\end{aligned}
$$

where

$$
\alpha=\frac{A^{0}}{\kappa}, \quad \mathcal{C}_{u, s}=\frac{C_{u, s}}{\kappa}, \quad \psi_{u, s}=\frac{K_{d}^{u, s}}{\kappa}
$$


Table 1: Non-dimensional parameters used in the model

\begin{tabular}{ll} 
Symbol & Physical interpretation \\
\hline$C_{R}$ & $\begin{array}{l}\text { maximum (jamming) coverage in the 1 } \\
\left(C_{R}=0.74756 . .\right)\end{array}$ \\
$\delta$ & ratio of antigen diameter to antibody diameter \\
$\theta$ & total coverage, $0 \leq \theta \leq C_{R}$ \\
$\bar{\theta}$ & normalized total coverage, $0 \leq \bar{\theta} \leq 1$ \\
$\mathcal{B}_{u, s}$ & coverage by end-on/accessible side-on antibodies \\
$\mathcal{B}$ & active coverage (all accessible antibodies) \\
$\overline{\mathcal{B}}_{u, s}$ & normalized coverage by end-on/accessible side-on antibodies \\
$\overline{\mathcal{B}}$ & normalized active coverage (total) \\
$\kappa$ & maximum antibody concentration (jamming limit) \\
$\alpha$ & normalized antigen concentration \\
$\psi_{u, s}$ & normalized affinity constants for end-on/side-on antibodies \\
$\mathcal{C}_{u, s}$ & normalized reaction product (signal) resulting from \\
$\mathcal{C}$ & end-on/side-on antibodies \\
& total normalized reaction product (signal).
\end{tabular}

are the normalized antigen concentration, product concentrations and affinity constants, respectively. By substituting (7) into (8), we obtain a cubic equation which can be solved to yield first $\mathcal{C}_{s}$ and then $\mathcal{C}_{u}$. The total antigen bound to antibody is calculated as

$$
\mathcal{C}=\mathcal{C}_{u}+\mathcal{C}_{s}
$$

and used in our simulations as a measure of the assay signal. The non-dimensional parameters introduced above are summarized in Table 1.

For situations when there is no distinction between the affinities of end-on and side-on antibodies (provided they are active), an equivalent but simpler approach would be to use a single reaction

$$
A+B \underset{k^{\prime}}{\stackrel{k}{\rightleftarrows}} C,
$$

with associated differential equation

$$
\frac{d C}{d t}=k A(t)\left(B^{0}-C(t)\right)-k^{\prime} C(t),
$$

which, after using the conservation law $A(t)+C(t)=A^{0}$ and the non-dimensionalization procedure described above, yields the signal

$$
\mathcal{C}=\frac{1}{2}\left[\psi+\alpha+\overline{\mathcal{B}}-\sqrt{(\psi+\alpha+\overline{\mathcal{B}})^{2}-4 \alpha \overline{\mathcal{B}}}\right]
$$

(In these kinetically uniform cases we used the notation $k=k_{u}=k_{s}, k^{\prime}=k_{u}^{\prime}=k_{s}^{\prime}, \psi=\psi_{u}=$ $\psi_{s}$, etc.)

\section{Numerical simulations}

The signal (or bound antigen concentration) calculated from either (9) or (10) can now be plotted against the total antibody coverage $\bar{\theta}$ in order to assess how the surface density 
affects the assay behaviour. A similar analysis, where $\mathcal{C}$ is considered a function of antigen concentration, $\alpha$, and used to generate theoretical dose-response curves, will be the subject of future work.

\subsection{Discussion of model parameters}

We now give a brief discussion of parameter values used for the numerical simulations.

Antibody orientation, $P_{\text {up }}, P_{\text {side }}$.

The percentages of antibodies adsorbed in the "up" and "side" configurations are known to vary with surface coverage: experimental evidence suggests that antibodies are more likely to lie flat at low surface coverage while crowding may favour an upward orientation, $[1,13,15]$. However, for simplicity, we assume here that $P_{\text {up }}$ and $P_{\text {side }}$ are constant throughout the deposition process. (Numerical simulations with nonconstant probabilities were presented in [9] but the corresponding signal curves did not display any significantly different behaviour.) Exact values for the antibody orientation parameters $P_{\text {up }}$ and $P_{\text {side }}$ are difficult to determine from practical information but we can study various theoretical possibilities and their outcomes. These are summarized in Table 2 and can be described as follows: "Side 1" and "Side 2 " refer to cases when the side-on particles are prevalent, thus $90 \%$ of antibodies are found in this configuration at all adsorption stages. These two cases are further distinguished by the percentage of antibodies adsorbing in the other configurations: $9 \%$ (and 5\%, respectively) have the end-on orientation, which implies that the remaining $1 \%$ (or $5 \%$, respectively) are head-on and thus unaccessible. Note also that the "Up 1" case describes the most desirable

Table 2: Various orientation possibilities used for the numerical simulations. The values $P_{\text {up }}$ and $P_{\text {side }}$ indicate the percentages of antibodies in the end-on and side-on configurations, respectively.

\begin{tabular}{lll}
\hline Side 1: & Mixed 1: & Up 1: \\
$P_{\text {up }}=0.09$ & $P_{\text {up }}=0.45$ & $P_{\text {up }}=0.81$ \\
$P_{\text {side }}=0.9$ & $P_{\text {side }}=0.5$ & $P_{\text {side }}=0.1$ \\
\hline Side 2: & Mixed 2: & Up 2: \\
$P_{\text {up }}=0.05$ & $P_{\text {up }}=0.25$ & $P_{\text {up }}=0.45$ \\
$P_{\text {side }}=0.9$ & $P_{\text {side }}=0.5$ & $P_{\text {side }}=0.1$ \\
\hline
\end{tabular}

situation when the end-on orientation predominates, while the "Mixed 2" case assigns equal adsorption probabilities to the four orientations possible in this one-dimensional model (up, down, left and right, as illustrated in Fig 1). Other orientation probabilities can be easily added to the study; however, we believe that the cases shown here represent a wide enough sample of all typical immobilization configurations.

Antibody affinity, $\psi_{u}, \psi_{s}$.

As discussed in the Introduction, the binding activity of antibodies could be affected by immobilization, regardless of whether they are accessible to antigens or not. Since the extent of this affinity deterioration is not precisely known, we investigate the following three possibilities:

Case 1 (High affinity): All immobilized antibodies, regardless of orientation, retain normal binding activity, similar to that displayed in solution.

Case 2 (Low affinity): All immobilized antibodies, regardless of orientation, have reduced binding activity. 
Case 3 (Mixed affinity): The side-on immobilized antibodies have reduced binding activity while the end-on antibodies retain their normal activity.

These possibilities are summarized in Table 3 below. It is difficult to calculate numerical values for the normalized affinity constants $\psi_{u}$ and $\psi_{s}$ based on real equilibrium dissociation constants described in the experimental literature, as we are employing an abstract 1-dimensional model (as discussed in the Introduction). Instead, the values 0.1 and 0.001 proposed in Table 3 are arbitrary affinity values that accurately capture the qualitative shift in the behaviour of signals. Values greater than 0.1 produce small signal curves due to the increased dissociation rate constant which results in a reduction of successfully bound antigen-antibody complexes. Furthermore, $\psi$ values less than 0.001 display signals similar to $\psi=0.001$ curves due to the inherent saturation effect.

Table 3: Different cases of antibody binding activity, based on affinity constants

\begin{tabular}{lll}
\hline $\begin{array}{l}\text { Case 1 } \\
\text { (High affinity) }\end{array}$ & $\begin{array}{l}\text { Case 2 } \\
\text { (Low affinity) }\end{array}$ & $\begin{array}{l}\text { Case 3 } \\
\text { (Mixed affinity) }\end{array}$ \\
\hline$\psi_{u}=\psi_{s}=0.001$ & $\psi_{u}=\psi_{s}=0.1$ & $\psi_{u}=0.001, \psi_{s}=0.1$ \\
\hline
\end{tabular}

Antigen size, $\delta$.

The size of the antigen relative to the detection antibody can vary greatly between assays and it has been shown to significantly affect the complex formation, [15]. In our study, we consider two possibilities: $\delta=1$ (corresponding to large antigens assumed, for simplicity, to have the same diameter as the antibody) and $\delta=0.1$ (small antigens). Recall that, in the RSA framework discussed in Section 2, the adsorbed particles (antibodies) have unit length.

Antigen concentration, $\alpha$.

As the signal is studied as a function of immobilized antibody density, the antigen concentration is assumed constant. We use the values $\alpha=0.5$ (low) and $\alpha=5$ (high) for the normalized antigen concentration, which correspond to antigen concentrations equal to half and five times, respectively, the maximum immobilized antibody concentration. (See Table 1.)

\subsection{Effect of antibody orientation and affinity on the assay signal}

The first set of simulations compares the assay signals when the antibody orientation on the surface varies according to the six possibilities presented in Table 2. This analysis is repeated for all three affinity cases (high, low and mixed), two different antigen sizes (small and large) as well as two different antigen concentrations (low and high). The results are presented in Figures 2-4. Note that the signals (or normalized reaction products $\mathcal{C}$ ) are given here as functions of the total antibody coverage, $\bar{\theta}$, and not just the functional antibody fraction. The maximum value on the horizontal axis, $\bar{\theta}=1$, represents the normalized jamming limit $\left(C_{R}=0.74756 .\right.$. , in the one-dimensional RSA formulation) which, for practical purposes, can be interpreted as a fully packed monolayer.

It is seen that for large antigens, different orientations of immobilized antibodies yield a wide range of signal shapes and amplitudes, while smaller antigens lead to less variety in the assay behaviour. This can be explained by noting that smaller antigens have better accessibility so immobilized side-on antibodies are thus more likely to be active, at both low and high surface coverage. Of significant practical interest is the "hooked" response 


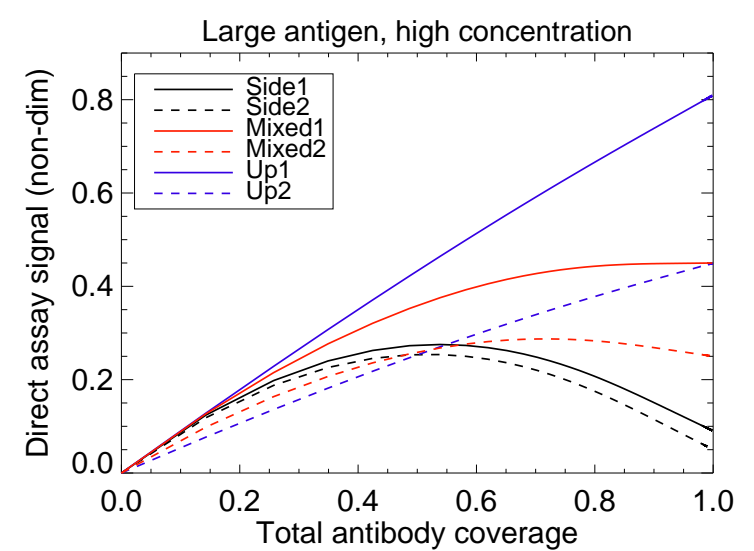

(a) $\delta=1, \alpha=5$

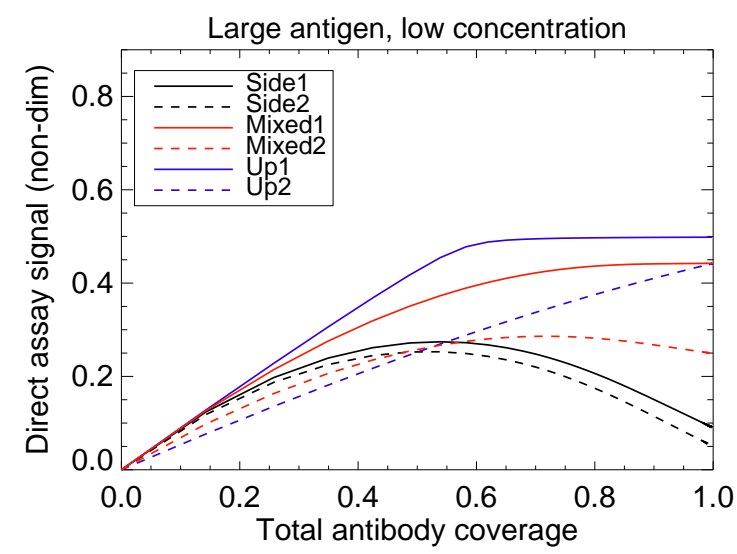

(c) $\delta=1, \alpha=0.5$

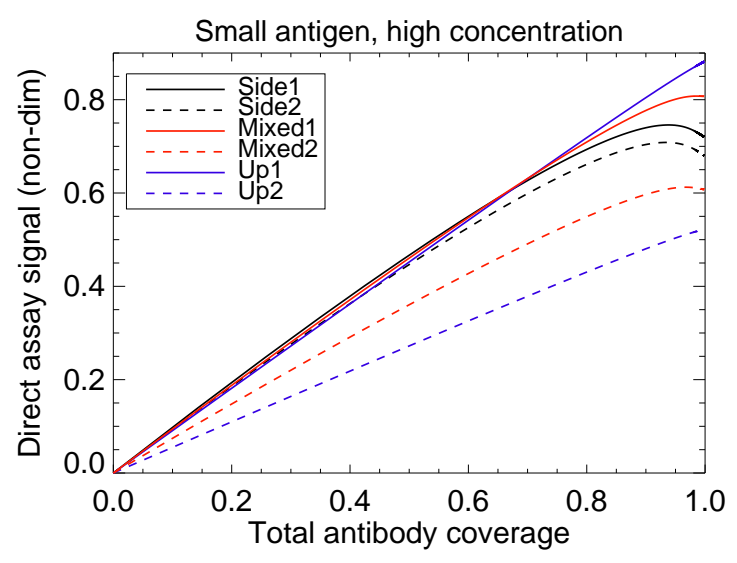

(b) $\delta=0.1, \alpha=5$

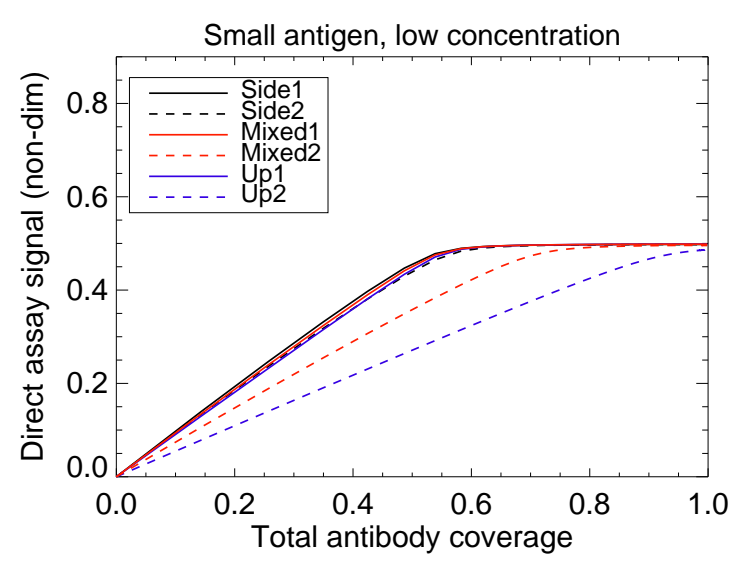

(d) $\delta=0.1, \alpha=0.5$

Figure 2: Comparison of different antibody orientations for Case 1 (high affinity, $\psi_{u}=\psi_{s}=$ $0.001)$, for both small $(\delta=0.1$, right $)$ and large $(\delta=1$, left $)$ antigens, at high $(\alpha=5$, top panels) and low ( $\alpha=0.5$, bottom panels) concentrations.

(signal decline at high antibody concentrations), which possesses a well-defined maximum value corresponding to optimal surface coverage. Our simulations suggest that hook curves occur when the side orientation predominates and the effect is more pronounced for large antigens. (For small antigens, the signal decline happens later, near maximal coverage, and is less significant). Similar qualitative behaviour has been widely reported in the experimental literature, $[1,2,11,17]$. For example, the results in [2] show that antigen-binding rates reach a maximum value when antibody coverage is low and a decrease in binding activity is observed at higher antibody concentrations. A flat-on orientation of antibodies is determined using neutron reflection (NR) measurements and the antigen used in this work is human chorionic gonadotrophin (hCG), characterized as a large, elongated molecule. Such an experimental configuration would correspond, in our simulations, to the parameter values associated with the side orientation (black) curves in Figures 2(a), 3(a) or 4(a). By contrast, linearly increasing curves (which is the desirable qualitative signal behaviour) are associated with excess antigen and either small antigens or large antigens with favourable (Up) orientation.

The second set of simulations (shown in the Supplementary Information, Figures S1-S3) 


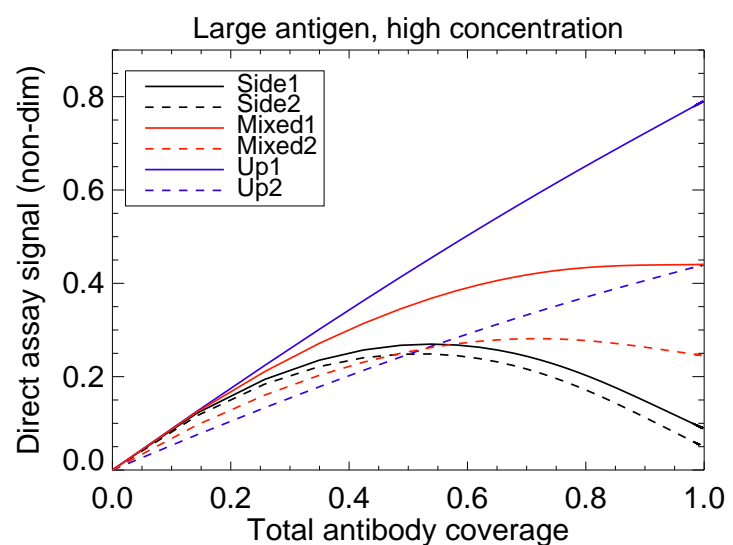

(a) $\delta=1, \alpha=5$

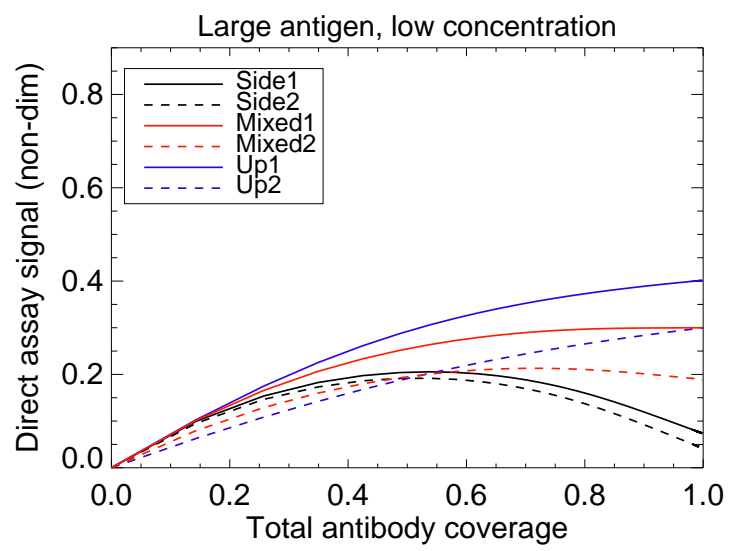

(c) $\delta=1, \alpha=0.5$

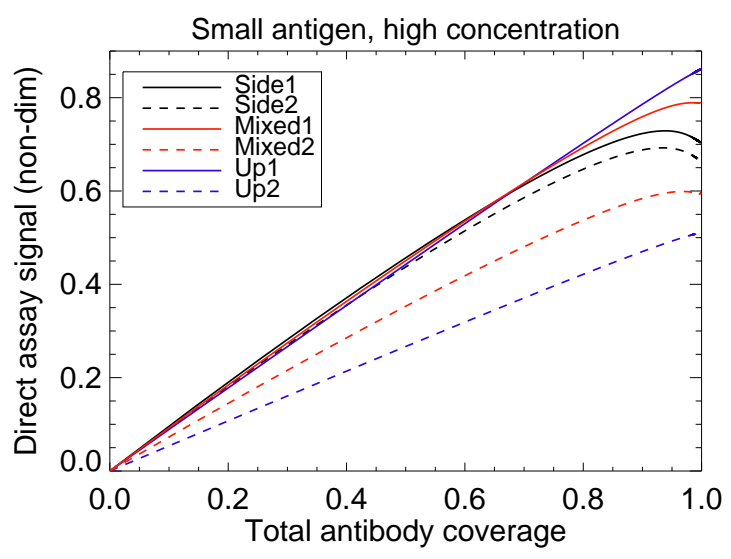

(b) $\delta=0.1, \alpha=5$

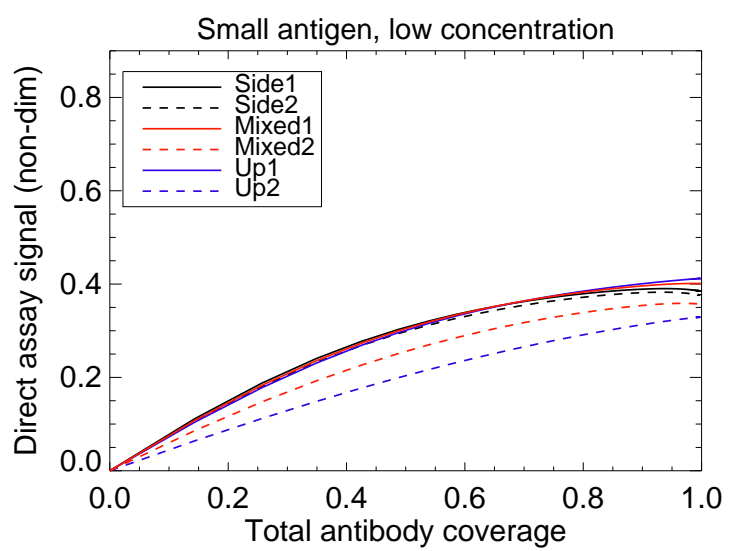

(d) $\delta=0.1, \alpha=0.5$

Figure 3: Comparison of different antibody orientations for Case 2 (low affinity, $\psi_{u}=\psi_{s}=$ $0.1)$, for both small ( $\delta=0.1$, right) and large $(\delta=1$, left $)$ antigens, at high $(\alpha=5$, top panels) and low ( $\alpha=0.5$, bottom panels) concentrations.

compare the signal curves associated with different antibody binding abilities. From these simulations (and similar ones with different $\alpha$ values) it appears that assigning different binding abilities to antibodies has no effect on the signal shape or strength at high antigen concentrations and only a small effect at low concentrations. This is not surprising as, in the case of excess antigen, the amount that is bound will be limited by the available antibody and hence the corresponding (solid) curves in Figures S1-S3 also represent the fraction of active antibody coverage.

Another observable difference between the various kinetic abilities studied above is the flattening of the signal curves near jamming, which occurs at low antigen concentrations when at least some of the antibodies retain high activity (Figures 2 and 4, corresponding to Case 1 and Case 3), but not when the affinity is uniformly low (Figure 3, Case 2). The presence of such signal saturation states can be explained by insufficient antigen under favourable antibody operating conditions (good affinity and orientation).

Finally, comparing the two sets of simulations shown in Figures 2-4 and Figures S1-S3, our model suggests that antibody orientation (and therefore accessibility of binding sites) has more 


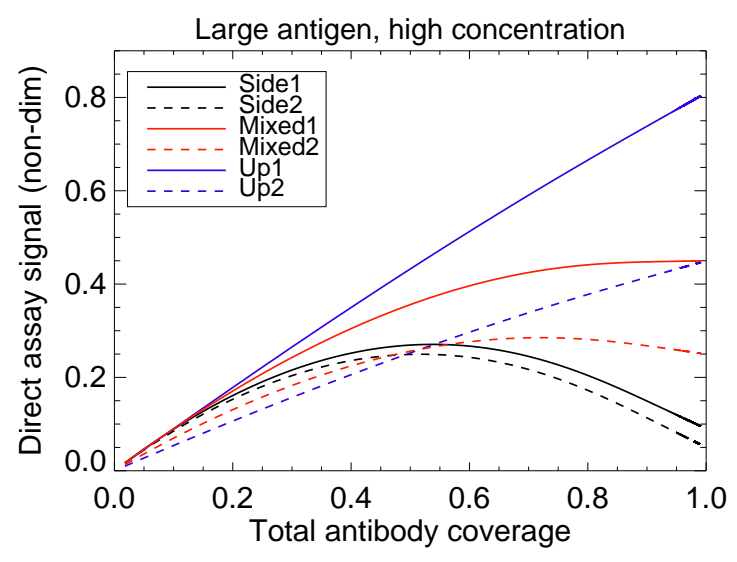

(a) $\delta=1, \alpha=5$

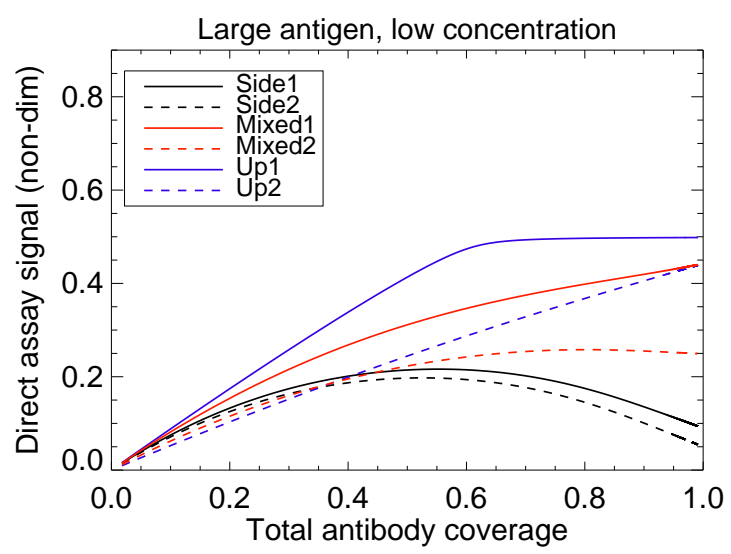

(c) $\delta=1, \alpha=0.5$

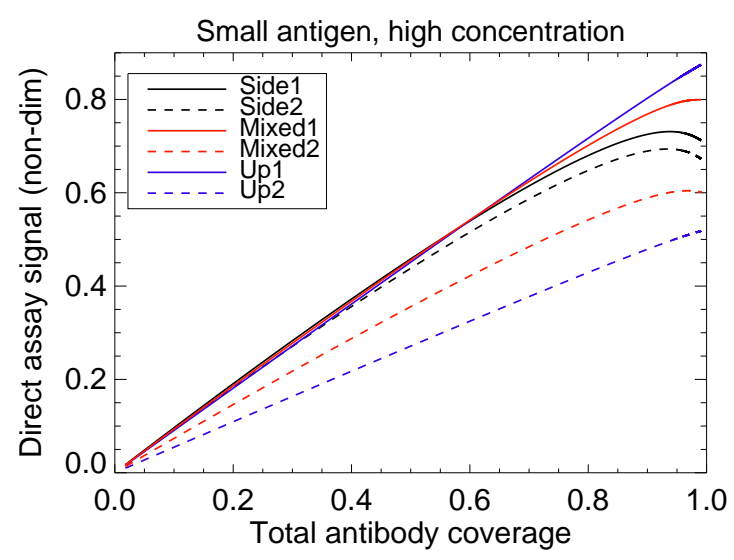

(b) $\delta=0.1, \alpha=5$

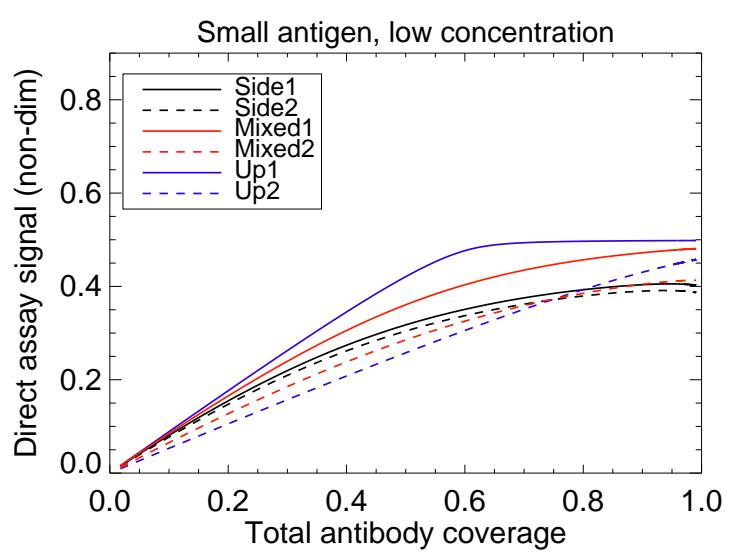

(d) $\delta=0.1, \alpha=0.5$

Figure 4: Comparison of different antibody orientations for Case 3 (mixed affinity, $\psi_{u}=0.001$, $\left.\psi_{s}=0.1\right)$, for both small ( $\delta=0.1$, right $)$ and large $(\delta=1$, left $)$ antigens, at high $(\alpha=5$, top panels) and low ( $\alpha=0.5$, bottom panels) concentrations.

impact on the assay behaviour than its affinity (especially at high antigen concentrations). A similar conclusion was reached in [18] where the binding between hCG and immobilized anti-hCG was studied on various surface types and the antigen-antibody ratio was found to be affected mainly by orientation rather than decrease in antibody bioreactivity.

\section{Conclusions}

We have presented a mathematical model which describes antibody adsorption and distribution on a solid surface using a one-dimensional RSA framework (where particle diameters are equivalent to intervals filling a line) and estimates the fraction of functional antibodies as a function of surface coverage, using parameters such as orientation probability and size of antigen. The total amount of antigen bound (or assay signal) is then theoretically predicted for various orientation configurations and degrees of affinity loss.

We note that this modelling strategy deals with features common to many different assays 
rather than focussing on a specific case study. For example, surface properties (charge, hydrophobicity, etc.) and bulk concentration of antibodies are known to affect their adsorption and orientation [13] and are reflected by the $P_{u p}, P_{\text {side }}$ parameters, while particular choices of the antibody-antigen pair are described by $\delta, \alpha, \psi$, etc. Various published experimental studies corresponding to specific choices of these parameters were cited throughout the paper to complement our theoretical simulations.

Many studies $[14,5,16]$ have shown that assays using well-oriented antibodies (achieved, for example, with bioaffinity immobilization techniques) exhibit better binding efficiency and sensitivity compared to randomly oriented ones. Although orientation of antibodies is known to affect their affinity, in this modelling framework we have considered these properties independently and shown that orientation has a more pronounced effect on complex formation than affinity. Thus, our model suggests that the poor performance displayed by randomly immobilized particles results mainly from reduced accessibility and, to a lesser extent, from impaired binding ability.

In order to facilitate the analytical and numerical study of this model, the geometric and kinetic features of the assay were greatly simplified. For example, only three different antibody orientations were considered (up, down, side) and the "Up" antibodies were assumed to be unconditionally accessible. Moreover, the immobilized particles were assumed rigid in this framework, with no possibility of further movement, shape change or overlap. These restrictions will be removed in future studies and there is huge potential for modelling other adsorption and transport effects, as sensors often rely on microfluidic elements and particle immobilization on spherical surfaces. In spite of the simplifying assumptions mentioned above, the model gives good qualitative agreement with experimental observations and we can conclude that a theoretical approach to assay optimization, which quantifies the effect of various design parameters on the signal curves, is feasible and adaptable to many physical settings.

It is still common practice in immunoassay preparation to adsorb antibodies onto the solid surface until saturation is reached as it is believed that a higher density would reduce the possibility of structural unfolding and yield better performance (see comments in [2]). However, practical studies have reported seemingly contradictory situations where densely packed antibodies were either seen to improve antigen binding as a consequence of favourable outward orientation [1], or else decrease it due to steric hindrance effects [17]. Our theoretical model shows that the assay behaviour at high antibody densities depends on many system parameters such as antibody orientation, relative size and concentration of antigen, etc. and therefore a different design approach is needed in each of the cases arising from this analysis.

\section{Conflicts of interest}

There are no conflicts to declare.

\section{Acknowledgements}

E. Kelly gratefully acknowledges financial support from the Irish Research Council, GOIPG/2013/812.

R. Nooney was supported by the Science Foundation Ireland under grant numbers 10/CE/B1821 and 14/TIDA/2334. 


\section{References}

[1] B. Saha, T.H. Evers and M.W.J. Prins, How antibody surface coverage on nanoparticles determines the activity and kinetics of antigen capturing for biosensing Anal. Chem., 86, 8158-8166 (2014).

[2] H. Xu, J.R. Lu, and D.E. Williams, Effect of surface packing density of interfacially adsorbed monoclonal antibody on the binding of hormonal antigen human chorionic gonadotrophin, J. Phys. Chem. B, 110, 1907-1914 (2006).

[3] D.C. Malaspina, G. Longo and I. Szleifer, Behaviour of ligand binding assays with crowded surfaces: Molecular model of antigen capture by antibody-conjugated nanoparticles, PLoS ONE, 12 (9):e0185518, https://doi.org/10.371/journal.pone.0185518 (2017).

[4] C. Dupont-Gillain, Orientation of adsorbed antibodies: in situ monitoring by QCM and random sequential adsorption modeling, Proteins at Interfaces III State of the art, T. Horbett et al., ACS Symposium Series, American Chemical Society (2012).

[5] N. Tajima, M.Takai and K. Ishihara, Significance of antibody orientation unraveled: welloriented antibodies recorded high binding affinity, Anal. Chem., 83, 1969-1974 (2011).

[6] J.W. Evans, Random and cooperative sequential adsorption. Rev. Mod. Phys., 651281 (1993).

[7] J.C. Roach, V. Thorsson and A.F. Siegel, Parking strategies for genome sequencing, Genome Research, 10, 1020-1030 (2000).

[8] A. Rényi, On a one-dimensional problem concerning random space-filling (In Hungarian). Publ. Math. Inst. Hung. Acad. Sci., 3, 109-127 (1958).

[9] D. Mackey, E. Kelly and R. Nooney, Modelling random antibody adsorption and immunoassay activity, Math. Biosci. Eng., 13, 6, 1159-1168 (2016).

[10] D. Mackey, E. Kelly and R. Nooney, A competitive random sequential adsorption model for immunoassay activity, Progress in Industrial Mathematics at ECMI 2016, Springer International Publishing (2018, to appear).

[11] X. Zhao, F. Pan, B. Cowsill, J.R. Lu, L. Garcia-Gancedo, A.J. Flewitt, G.M. Ashley and J. Luo, Interfacial immobilization of monoclonal antibody and detection of human prostate-specific antigen, Langmuir, 27, 7654-7662 (2011).

[12] P.L. Krapivsky, Kinetics of random sequential parking on a line. J. Stat. Phys., 69, 135-150 (1992).

[13] M.E. Wiseman and C.W. Frank, Antibody adsorption and orientation on hydrophobic surfaces, Langmuir, 28, 1765-1774 (2012).

[14] A.K. Trilling, J. Beekwilder and H. Zuilhof, Antibody orientation on biosensor surfaces: a minireview, Analyst, 138, 1619-1627 (2013).

[15] W. Schramm and S. Paek, Antibody-antigen complex formation with immobilized immunoglobulins, Anal. Biochem., 205, 47-56 (1992). 
[16] V. Gubala, C. Crean, R. Nooney, S. Hearty, B. McDonnell, K. Heydon, R. O’Kennedy, B.D. MacCraith, and D.E. Williams, Kinetics of immunoassays with particles as labels: effect of antibody coupling using dendrimers as linkers. Analyst, 136, 2533-2541 (2011).

[17] M.L.M. Vareiro, J. Liu, W. Knoll, K. Zak, D.E. Williams, A. Toby and A. Jenkins, Surface plasmon fluorescence measurements of human chorionic gonadotrophin: role of antibody orientation in obtaining enhanced sensitivity and limit of detection, Anal. Chem., 77, 2426-2431 (2005).

[18] S. Chen, L. Liu, J. Zhou and S. Jiang, Controllong antibody orientation on charged self-assembled monolayers, Langmuir, 19, 2859-2864 (2003). 